\title{
Identification and Local Chemical Analysis of Pharmaceutical Impurities
}

T.L. Nylese, $*$ R.A. Anderhalt, * U. Patel $* *$

* EDAX, a division of Ametek, 91 McKee Dr. Mahwah, NJ 07430

** New Jersey Institute of Technology, Newark, NJ 07102

The presence and amount of impurities in pharmaceutical drug products is regulated by various national and international agencies to ensure quality and efficacy of the manufactured drug product. Small amounts of degradation products or related-products of the active pharmaceutical ingredients (APIs) are allowable for the product to still be considered safe and effective [1]. The amount of these impurities is usually determined by a Quality Control sampling of product and analyzed using chromatographic techniques such as HPLC/MS to quantify that they are within the predetermined tolerable limits [2].

When Scanning Electron Microscopy (SEM) along with Energy Dispersive Spectroscopy (EDS) is employed to identify and characterize impurities in a drug product, information about the morphology and local environment surrounding the by-product can be attained and studied. Visual discrimination and chemical microanalysis with EDS can elucidate the size, shape and distribution of impurities of interest. Further, microanalysis that is spatially specific can provide a view of the chemical composition immediately surrounding the area wherein the altered product is formed, which can provide valuable insight to the mechanism of transformation. With an understanding of this process, manufacturers can take measures to decrease the likelihood of formation, increase product stability and therefore extend drug product shelf life.

In this work, SEM/EDS is used to study impurities in Iron Supplement tablets by identifying the chemistry of the API, $\mathrm{FeSO}_{4}$, and then using local spectral analysis to locate the presence of impurity products in the form of high iron, low sulfur containing particulates and gather morphological and chemical information, Figure 1 and Figure 2. A comparison of chemistry finds the presence of these particulates in slow release Iron supplement tablets, containing Calcium Carbonate as a primary excipient while rapid release Iron supplement tablets, containing Dicalcium Phosphate as a primary excipient, do not contain the particulates. While variations in calcium/iron ratios to sulfur are present in spectral analysis of discreet sampling areas, Figure 3, careful consideration of chemical maps must be done to account for matrix effects. Imaging and mapping techniques are used to show the chemistry of the surrounding area including the API, excipients and impurities, Figure 4.

References

[1] N. Rama Rao et al., Pharmaceutical Impurities: An Overview, Indian Journal of

Pharmaceutical Education and Research, 44(3), 301-310.

[2] Handbook of Stability Testing in Pharmaceutical Development: Regulation, Methodologies, and Best Practices (2009). New York, NY: Springer 


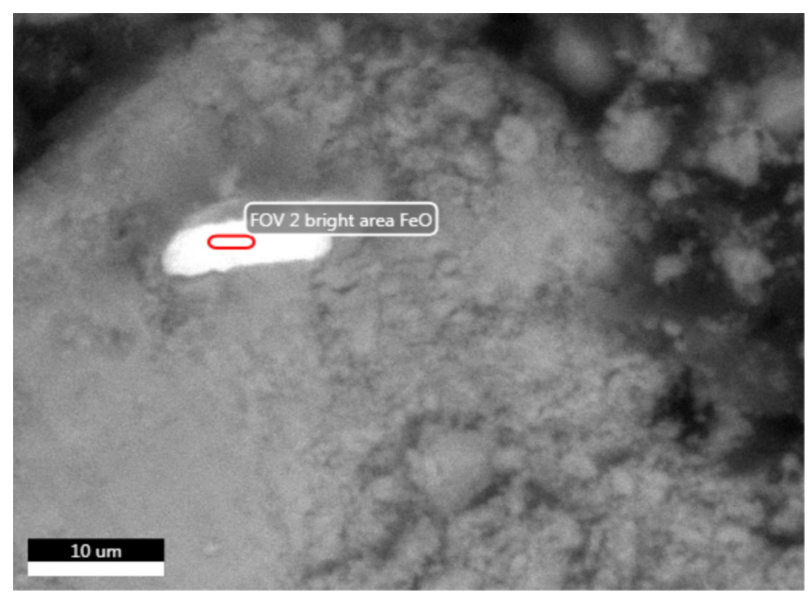

Figure 1: SEM Image, area of interest; bright particle is the impurity.

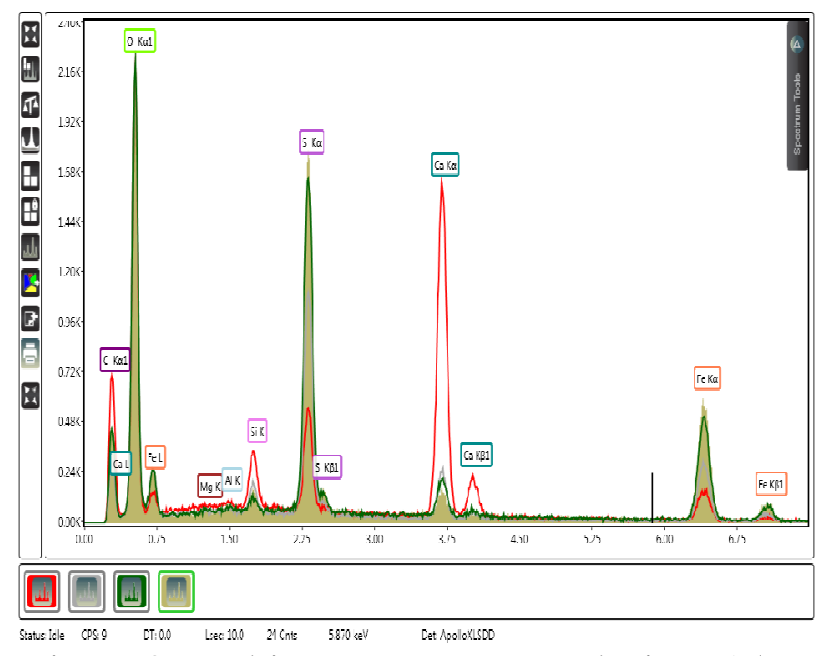

Figure 3: Multi-area spectrum analysis at 15 $\mathrm{kV}$ shows ratio changes of elements of interest $(\mathrm{Ca} / \mathrm{Fe} / \mathrm{S})$.

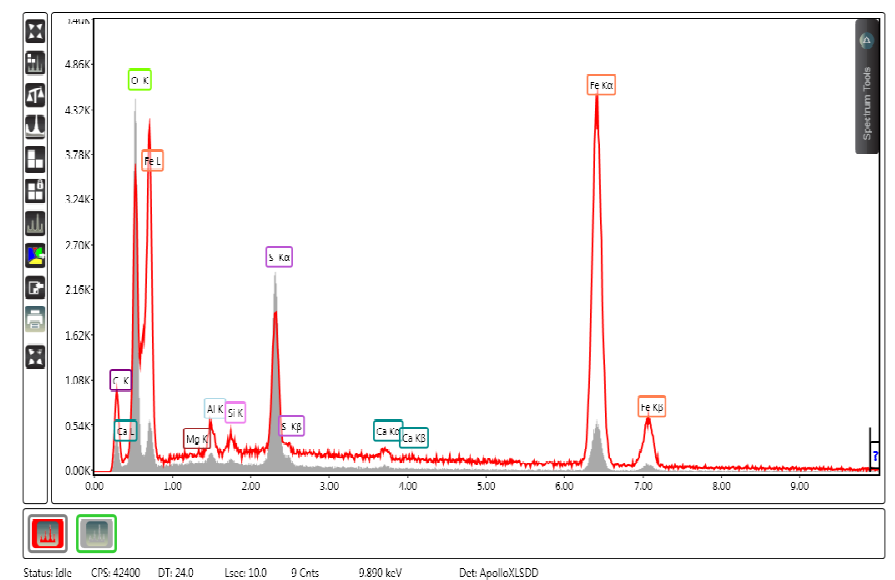

Figure 2: $15 \mathrm{kV}$ spectrum of the bright particle in outline showing higher iron peak intensity compared to $\mathrm{FeSO}_{4}$ in solid gray spectrum.

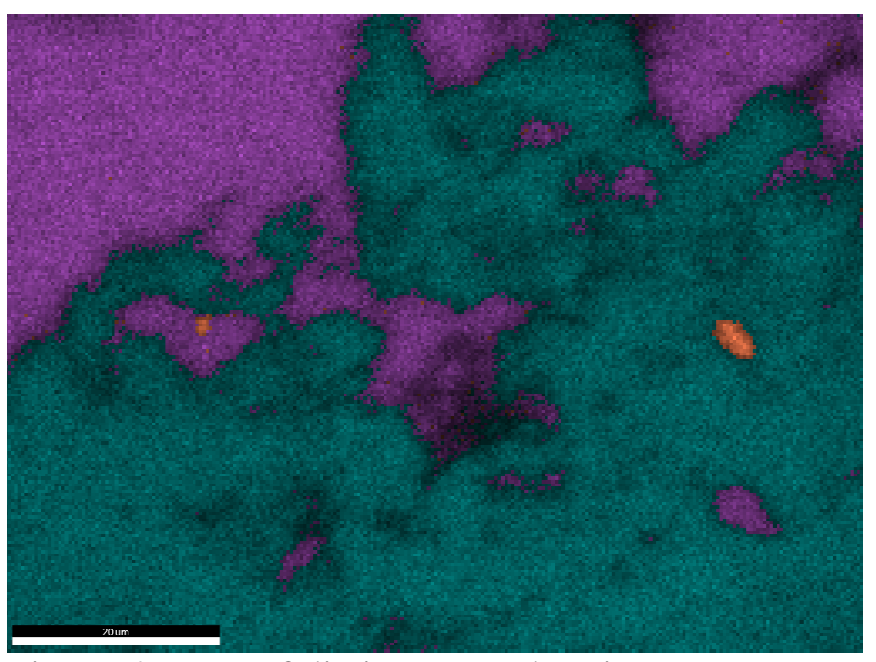

Figure 4: Map of distinct area showing two intense iron particulates (orange) relative to sulfur (purple). Calcium in green. 